\title{
IDENTIFICATION OF AN S-ANTIGEN-LIKE MOLECULE IN HUMAN CHOROID PLEXUS AND CEREBROSPINAL FLUID
}

\author{
H. S. DUA ${ }^{1}$, PATRICIO F. REYES ${ }^{2}$, JEFFERY A. BARRETT ${ }^{1}$, MICHAEL S. ABRAMS ${ }^{1}$, \\ ROLAND SCHWARTING ${ }^{3}$, CHERYL M. CRAFT $^{4}$, LARRY A. DONOSO $^{1}$ \\ Philadelphia and Dallas, USA
}

\begin{abstract}
SUMMARY
Sensitisation to retinal S-antigen has been implicated in the pathogenesis of several clinical forms of posterior uveitis. S-antigen-like molecules have recently been demonstrated in the brain and choroid plexus of experimental animals. We used a panel of four monoclonal antibodies (MAbs), MAbF4-C1, MAbC10-C10, MAbA2-G5 and MAbA9-C6, which define specific epitopes in the amino, mid and carboxyl terminal portions of $S$-antigen in order to identify an S-antigen-like molecule in human choroid plexus and cerebrospinal fluid (CSF). Three MAbs, MAbF4-C1, MAbC10-C10 and MAbA9-C6, localised an S-antigen-like molecule to the cytoplasm of the epithelial cells of the human choroid plexus. Polymerase chain reaction of cDNA from choroid plexus verified the presence of S-antigen homologues in the choroid plexus. The presence of an $\mathrm{S}$-antigen-like molecule in the CSF was demonstrated by western blots in seven CSF samples from patients with a variety of neuropathological disorders. It is proposed that immunological cross-reactivity and biochemical similarity between retinal S-antigen and an S-antigen-like molecule in human choroid plexus and CSF could form a basis for neurological manifestations observed in certain clinical forms of uveitides.
\end{abstract}

Experimental autoimmune uveitis (EAU) is a T-helper/ inducer cell $(\mathrm{CD} 4+)$ mediated disease of the retina and uveal tract of the eye. ${ }^{1,2}$ EAU can be induced by immunisation of animals with several retinal proteins. ${ }^{3,4}$ Retinal $\mathrm{S}$-antigen-induced EAU is regarded as an excellent animal model of human posterior uveitis as it closely parallels all the clinical and histopathological changes observed in humans. ${ }^{1}$ Retinal S-antigen is a $48 \mathrm{kDa}$ protein present

From ${ }^{1}$ Retina Service Wills Eye Hospital, Philadelphia; ${ }^{2}$ Department of Neurology and the Alpers Neuropathology Laboratory and ${ }^{3}$ Department of Immunopathology, Jefferson Medical College, Thomas Jefferson University, Philadelphia; ${ }^{4}$ Laboratory for Molecular Genetics, University of Texas Health Science Center, Dallas, USA.

Correspondence to: Larry A. Donoso, MD, PhD, Research Division, Wills Eye Hospital, 900 Walnut Street, Philadelphia, PA 19107, USA. predominantly in the rod photoreceptors and in pinealocytes of the pineal gland. ${ }^{5,6}$ Although it has been used extensively in the study of EAU its exact physiological role in the eye is unknown. There is evidence to indicate that it participates in phototransduction - the biochemical mechanism by which light energy is converted to a nerve impulse. S-antigen interacts with an enzyme, cyclic GMPase, to 'arrest' the amplified phototransduction cascade. ${ }^{78}$ Biochemical analysis of S-antigen has revealed that the molecule is made up of 404 amino acids. ${ }^{9}$ Several immunologically active peptides have been characterised within the molecule $e^{10-12}$ and antibodies that bind to specific peptides have been generated. ${ }^{10-13}$

In recent years it has been demonstrated in animals that retinal S-antigen-specific monoclonal antibodies crossreact with protein(s) in non-photoreceptive tissues, particularly nervous tissue and choroid plexus, ${ }^{14.15}$ indicating the presence of retinal S-antigen-like molecules in these tissues. Lohse et al. ${ }^{16}$ have characterised a bovine brain homologue of S-antigen from cDNA clones. This molecule, termed $\beta$-arrestin, is slightly larger (418 amino acids) than retinal S-antigen but shows a 59\% amino acid sequence identity with retinal S-antigen.

As some forms of human uveitides, such as Behçet's disease, ${ }^{17}$ sympathetic ophthalmitis ${ }^{18}$ and Vogt-Koyanagi-Harada's (VKH) syndrome, ${ }^{19}$ show evidence of neurological involvement, the possibility of immunological cross-reactivity between the eye and the nervous system as a basis for such involvement appears likely and warrants further investigation. We used a panel of four anti-retinal S-antigen-specific monoclonal antibodies, that bind to peptides located at the amino, mid, and carboxyl regions of the S-antigen molecule, to identify $\mathrm{S}$-antigen-like molecule(s) in the human choroid plexus and cerebrospinal fluid (CSF). We also employed the polymerase chain reaction technique to further characterise the S-antigen-like molecule in human choroid plexus. 


\section{MATERIALS AND METHODS}

\section{S-Antigen and Monoclonal Antibodies}

$\mathrm{S}$-antigen was purified from bovine retinal homogenate by Sephadex gel chromatography using a modification of the method described previously. ${ }^{20}$

Four IgG mouse monoclonal antibodies (MAbs) designated MAbF4-C1, MAbC10-C10, MAbA2-G5 and MAbA9-C6, were used in the study. The general properties of these MAbs including the specific binding sites have been reported previously. ${ }^{11-13}$. MAbF4-C1 binds to the amino terminus, whereas MAbC10-C10 binds to the mid portion, and MAbA2-G5 and MAbA9-C6 bind to the carboxyl terminus of the molecule. MAbA2-G5 is specific for bovine $\mathrm{S}$-antigen.

\section{Immunohistochemistry}

Fixed tissue sections of normal human choroid plexus were obtained from the Alpers Neuropathology Laboratory (Thomas Jefferson University, Philadelphia, PA). The slides were deparaffinised, hydrated and treated to block endogenous peroxidase activity. The slides were then sequentially incubated with primary MAb, biotinylated anti-mouse immunoglobulin and avidin-biotin-horseradish peroxidase complex. Colour development was performed with diaminobenzidine; positive staining was indicated by a brown colour.

\section{SDS Polyacrylamide Gel Electrophoresis (PAGE) and western Blots}

Seven CSF samples were selected at random from the CSF bank of the Alpers Neuropathology Laboratory (Thomas Jefferson University, Philadelphia, PA). Age of the patients (3 females and 4 males) ranged from 70 to 90 years. Six patients had Alzheimer's disease and their CSF samples were normal on biochemical and microscopic examination. The seventh patient had Lyme's disease with meningitis and his CSF showed elevated proteins and

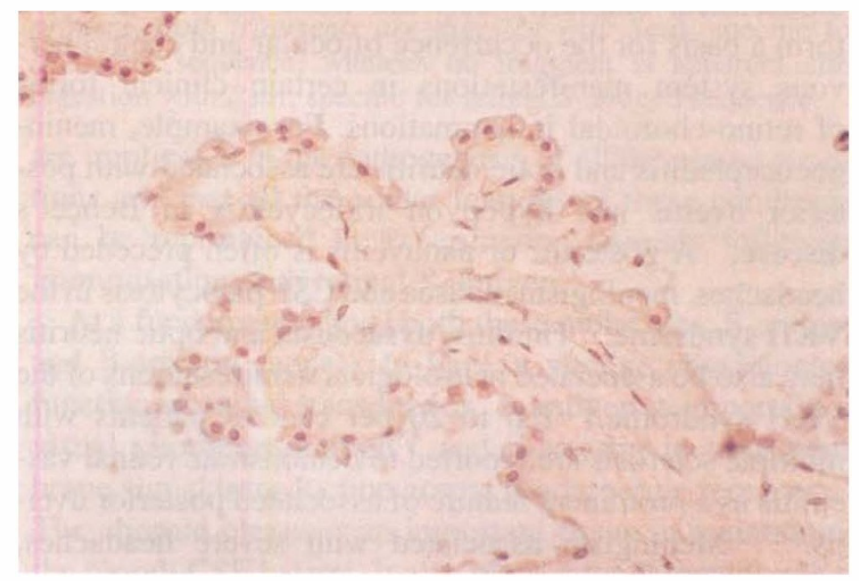

Fig. 1a lymphocytes (see legend to Fig. 2). Each of the seven CSF samples and control $\mathrm{S}$-antigen solution were subjected to SDS PAGE in duplicate, on an 8-25\% gradient SDS gel using the Pharmacia Phast system. One gel was stained using the silver staining method as described by the manufacturer (Pharmacia, Piscataway, NJ). Protein from the gel was blotted onto a nitrocellulose membrane and stained with each of the anti S-antigen MAbs using the alkaline phosphatase-anti-alkaline phosphatase technique. Details of SDS PAGE, western blotting and staining methods have been described before. ${ }^{21}$

\section{Polymerase Chain Reaction (PCR) Amplification with S-Antigen Homologue ( $\beta$-Arrestin) Primers and Complementary DNA (cDNA)}

Choroid plexus and retina cDNA were reverse transcribed with AMV reverse transcriptase from $1 \mu \mathrm{g}$ of total RNA extracted from choroid plexus and retina. Twenty cycles of PCR were used for amplification. After PCR, the samples were digested with restriction endonucleases for specific sites present in S-antigen (RsaI) or its homologue, $\beta$-arrestin $(\mathrm{SauI})$, as determined by direct sequencing of PCR-amplified controls. The samples were electrophoresed on 5\% polyacrylamide gel and exposed to X-ray film for 30 minutes. The bands developed on the autoradiogram were studied for the effect of endonuclease digestion on the PCR samples of choroid plexus and retina.

\section{RESULTS}

Three MAbs, MAbF4-C1, MAbC10-C10 and MAbA9-C6, localised S-antigen immunoreactivity to the human choroid plexus. Control slides stained with SP2/0 mouse myeloma supernatant were negative (Fig. 1a, b). In these experiments MAbF4-C1 showed the most intense stain and MAbA9-C6 the least. The staining pattern was localised to the cytoplasm of the epithelial cells of the choroid plexus. The stromal tissue did not stain. MAbA2-G5, bovine specific, did not stain human choroid plexus.

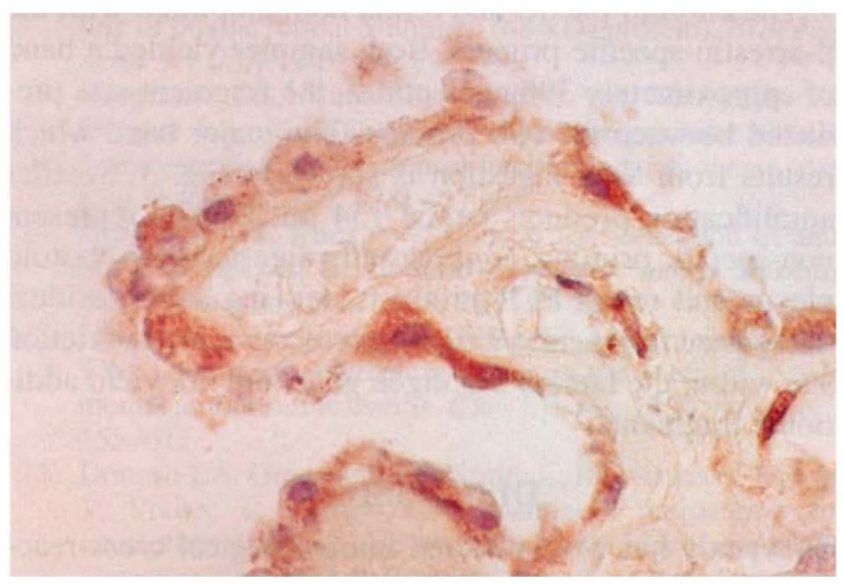

Fig. 1b

Fig. 1. (a) Photomicrograph of human choroid plexus villus showing absence of immunoreactivity with SP2/0 mouse myeloma supernatant. (Negative control, magnification $\times 120$.) (b) Photomicrograph of human choroid plexus villus showing marked immunoreactivity with MAbF4-C1. The apex of the villus has been magnified to illustrate marked immunoreactivity in the cytoplasm of the epithelial cells. (MAbF4-Cl indirect immunoperoxidase and haematoxylin, $\times 475$.) 


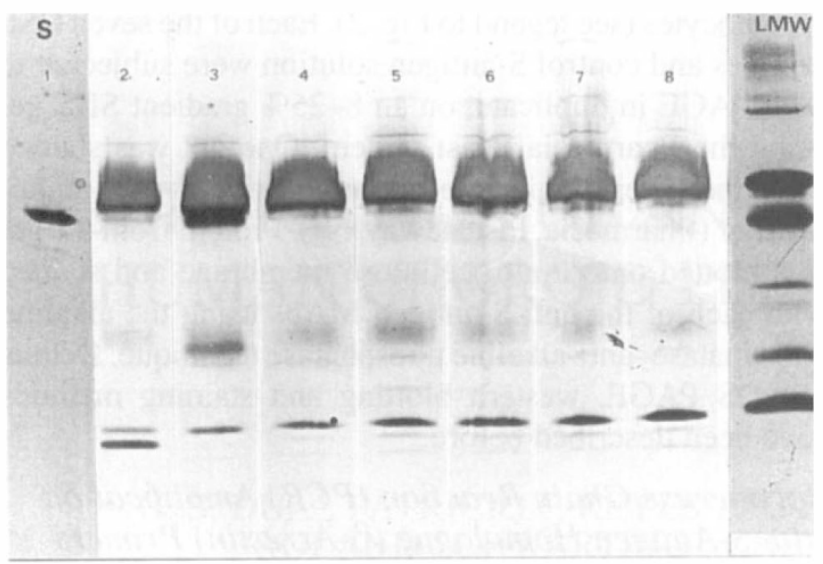

Fig. 2a

Fig. 2. (a) SDS PAGE of seven CSF samples (silver stained). Lane 1 represents purified bovine $S$-antigen and lanes 2 to 8 represent patient CSF samples. LMW, low molecular weight calibration kit proteins (a, phosphorylase $94 k ; b$, albumin $67 k$; , ovalbumin $43 k ; d$, carbonic anhydrase $30 k ; e$, trypsin inhibitor $20 k ; f$, $\alpha$-lactalbumin $14.4 k$ ). (b) Western blot of human CSF with monoclonal antibody MAbF4-C 1. Lane 1 represents purified bovine S-antigen and lanes 2 to 8 represent patient CSF samples corresponding to similarly numbered lanes of the gel in Fig. 1 (a). Arrow indicates position of authentic native S-antigen (48k). Lane ' 3 ' of the gel and western blot represents CSF sample of a male patient who had Lyme's disease with meningitis, elevated CSF proteins and lymphocytes. This lane has the most prominent band ofS-antigen-like molecule compared to the other lanes. All other patients had Alzheimer's disease with normal CSF.

Several protein bands were seen on SDS PAGE of the CSF samples. The electrophoresis profile of the seven CSF samples was similar but not identical (Fig. 2a). All three MAbs also detected an S-antigen-like molecule in western blots a human CSF as shown in Fig. 2b. In these experiments a molecule of approximately $48 \mathrm{kDa}$ which migrated slightly higher than authentic retinal S-antigen was observed. Similarly, MAbF4-C1 stained most intensely, reacting with all seven CSF samples tested, whereas MAbA9-C6 showed weak reactivity with four samples and reacted strongly with the remaining three samples. MAbA2-G5 did not react.

The choroid plexus and retina both amplified with the $\beta$-arrestin-specific primers. Both samples yielded a band of approximately 396 nucleotides, the fragment size predicted between the two primers. The major band which results from SauI digestion is shown in Fig. 3. Smaller amplification products below 154 nucleotides represent non-specific priming. SauI partially digested both choroid plexus and retina PCR products, leaving some residual undigested fragments. $R s a \mathrm{I}$, which recognises a restriction site within the human S-antigen gene, did not yield additional fragments.

\section{DISCUSSION}

This study has demonstrated immunological cross-reactivity between human ocular and central nervous system tissues. Three of the four monoclonal antibodies tested reacted with choroid plexus and CSF. The amino acid sequence of MAbA2-G5 binding site is unique to bovine $\mathrm{S}$-antigen and probably explains why this MAb did not stain positive in these cases. The binding of retinal S-anti- gen-specific monoclonal antibodies to protein(s) in the choroid plexus and CSF would indicate the presence of common antigenic epitopes. This has been further confirmed by endonuclease digestion of PCR products of choroid plexus and retina cDNA. The choroid plexus product could be digested with restriction endonuclease SauI which is specific for $\beta$-arrestin, and not with RsaI, specific for retinal $S$-antigen. This strongly suggests that the $S$-antigen-like molecule in human choroid plexus is $\beta$-arrestin. Both S-antigen and $\beta$-arrestin, however, belong to the same family of molecules and share a 59\% amino acid sequence homology. ${ }^{16}$ The immunological and biochemical similarity between these molecules could form a basis for the occurrence of ocular and central nervous system manifestations in certain clinical forms of retino-choroidal inflammations. For example, meningoencephalitis and optic neuritis are associated with posterior uveitis and hypopyon iridocyclitis in Behçet's disease. ${ }^{17} \mathrm{~A}$ posterior or panuveitis is often preceded by headaches, meningism, nausea and CSF pleocytosis in the VKH syndrome. ${ }^{22}$ Tinnitus, dysacousia and optic neuritis may also be associated neurological manifestations of the VKH syndrome..$^{22}$ Ten to 20 per cent of patients with multiple sclerosis are reported to demonstrate retinal vasculitis as a prominent feature of associated posterior uveitis. $^{23-25}$ Meningism associated with severe headaches, frank meningitis and electroencephalographic changes have been reported in some cases of sympathetic ophthalmitis. ${ }^{23}$ One report goes as far as stating that $50 \%$ of patients with uveal inflammation show some involvement of the central nervous system. ${ }^{23}$ It is interesting that apart from viral infection, (auto)immune-mediated mechanisms 


\section{Choroid Plexus}

\section{Retina}

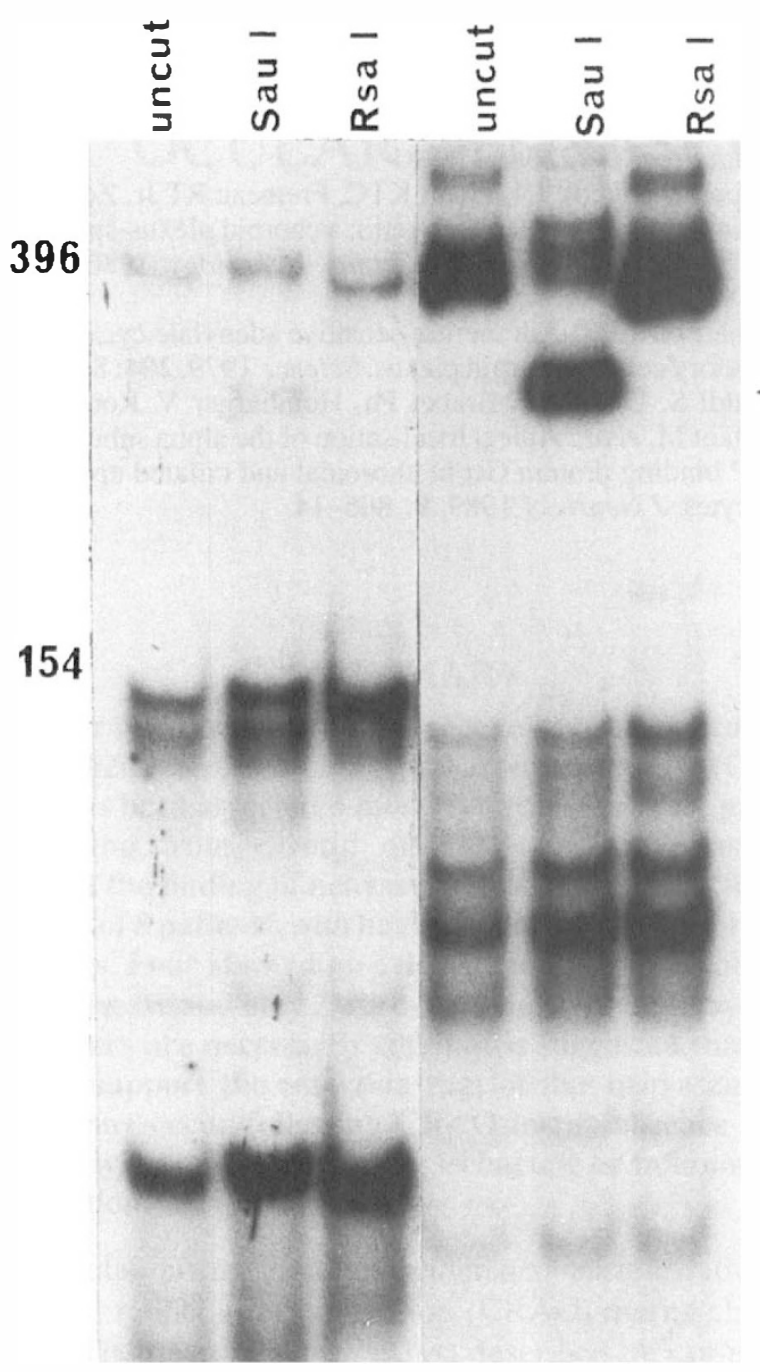

Fig. 3. Restriction endonuclease digestion of choroid plexus and retina cDNA fragments amplified with $\beta$-arrestin-specific primers. Both fragments are digested with SauI, specific for $\beta$-arrestin sequence, whereas no fragment is apparent after digestion with $R s a \mathrm{I}$, specific for retinal S-antigen sequence.

are implicated in the pathogenesis of all the above conditions and that all the ocular features of these conditions can be reproduced in experimental animals following immunisation with retinal S-antigen. ${ }^{1}$

At a fundamental level both these molecules, $S$-antigen and $\beta$-arrestin, appear to have a similar physiological function of signal transduction. S-antigen is important in visual phototransduction ${ }^{7,8}$ and $\beta$-arrestin in transmembrane signal transduction across $\beta$-adrenergic receptors. ${ }^{16}$ The choroid plexus is an important tissue in maintaining the blood-CSF barrier. It also plays a fundamental role in the synthesis and secretion of CSF proteins. ${ }^{26}$ This function is controlled in part by several signal transduction systems including the $\beta$-adrenergic-adenyl cyclase system. ${ }^{27}$ Brain G-protein, which is also necessary in the signal transduction process, has been localised immuno- histochemically to the apical processes of choroid plexus cells. ${ }^{28}$ It is quite likely, therefore, that $\beta$-arrestin is also located at this site. Future studies will determine whether the $S$-antigen-like molecule, $\beta$-arrestin, is actively synthesised and secreted into the CSF by the choroid plexus and whether its levels in the CSF are useful clinically, in monitoring any specific disease process.

Supported in part by the Retina Service, Wills Eye Hospital; National Institutes of Health grant EY5095; The Pennsylvania Lions Sight Conservation and Eye Research Foundation; the Crippled Children's Vitreoretinal Research Foundation (David Meyer, MD, Director); Research to Prevent Blindness Inc; and the Grampian Health Board, Aberdeen, Scotland (H. S. Dua).

Key words: $\beta$-adrenergic receptor, Cerebrospinal fluid, Human choroid plexus, Monoclonal antibody, S-antigen, Signal transduction.

\section{REFERENCES}

1. Forrester JV, Liversidge J, Dua HS, Towler H, McMenamin PG: Comparison of clinical and experimental uveitis. Curr Eye Res 1990, 9 (Suppl.): 75-84.

2. Mochizuki M, Kuwabara T, McAllister C, Nussenblatt RB, Gery I: Adoptive transfer of experimental autoimmune uveoretinitis in rats. Invest Ophthalmol Vis Sci 1985, 26: 1-9.

3. Faure JP: Autoimmunity and the retina. Curr Top Eye Res 1980, 2: 215-301.

4. Dua HS, Lee RH, Lolley R, Barrett JA, Abrams M, Forrester JV, Donoso LA: Induction of experimental autoimmune uveitis by the retinal photoreceptor cell protein, phosducin. Curr Eye Res 1992, 11 (Suppl): 107-11.

5. Kalsow CM and Wacker WB: Pineal reactivity of antiretina sera. Invest Ophthalmol Vis Sci 1977, 16: 181-4.

6. Mirshahi M, Faure JP, Brisson P, Falcon J, Guerlotte J, Collin JP: S-antigen immunoreactivity in retinal rods and cones and pineal photosensitive cells. Biol Cell 1984, 52: 195-8.

7. Kuhn H, Hall SW, Wilden U: Light-induced binding of 48$\mathrm{kDa}$ protein to photoreceptor membranes is highly enhanced by phosphorylation of rhodopsin. FEBS Lett 1984, 176: 473-8.

8. Zuckerman $\mathrm{R}$ and Cheasty JE: A $48 \mathrm{kDa}$ protein arrests cGMP phosphodiesterase activation in retinal rod disk membrane. FEBS Lett 1986, 207: 35-41.

9. Shinohara T, Dietzschold B, Craft CM, Wistow G, Early JJ, Donoso LA, Howitz J, Tao R: Primary and secondary structure of bovine retinal S-antigen (48-kDa protein). Proc Natl Acad Sci USA 1987, 84: 6975-9.

10. Gregerson DS, Fling SP, Obritsch WF, Merryman CF, Donoso LA: Identification of T-cell recognition sites in S-antigen: dissociation of proliferative and pathogenic sites. Cell Immunol 1989, 123: 427-40.

11. Gregerson DS, Knospe V, Donoso LA: Selection of antibody epitopes in an immuno-pathogenic neural autoantigen. J Neuroimmunol 1989, 24: 191-206.

12. Donoso LA, Gregerson DS, Fling SP, Merryman CF, Sery TW: The use of synthetic peptides in the study of experimental autoimmune uveitis. Curr Eye Res 1990, 9 (Suppl.): 155-61.

13. Donoso LA, Gregerson DS, Smith L, Robertson S, Knospe V, Vrabec T, Kalsow CM: S-antigen: preparation and characterisation of site-specific monoclonal antibodies. Curr Eye Res 1990, 9: 343-55.

14. Oliver J, Hewrbute S, Mirshahi M, Faure JP, Brisson P, Collin JP: Identification d'une proteine apparentée à l'antigene-S dans les plexus choroides de la Caille. CR Acad Sci Paris 1987, 305: 485-91.

15. Faure JP and Mirshahi M: S-antigen in non-ocular tissues. Curr Eye Res 1990, 9 (Suppl.): 163-7. 
16. Lohse ML, Benovic JL, Codine J, Caron MG, Lefkowitz JL: $\beta$-Arrestin: a protein that regulates $\beta$-adrenergic receptor function. Science 1990, 248: 1547-50.

17. Michelson J and Chisari FV: Behçet's disease. Surv Ophthalmol 1982, 26: 190-203.

18. Marak G: Recent advances in sympathetic ophthalmia. Surv Ophthalmol 1979, 24: 141-56.

19. Ohno S, Char D, Kimura S, O'Connor G: Vogt-KoyanagiHarada syndrome. Am J'Ophthalmol 1977, 83: 735-40.

20. Wacker WB, Donoso LA, Kalsow CM, Yankeelov JA Jr, Organisciak DT: Experimental allergic uveitis: isolation, characterization and localization of a soluble uveitopathogenic antigen from bovine retina. J Immunol 1977, 119: 1949-58.

21. Dua HS, Hossain P, Brown PAJ, McKinon A, Forrester JV, Gregerson DS, Donoso LA: Structure function studies of S-antigen: use of proteases to reveal a dominant uveitogenic site. Autoimmunity 1991, 10: 153-63.

22. Lightman $S$ and Chan CC: Immune mechanisms in choroidoretinal inflammation in man. Eye 1990, 4: 345-53.
23. Duke-Elder S and Perkins ES: Uveitis associated with diseases of the central nervous system and uveitis of unknown etiology. In: Duke-Elder S, ed. System of Ophthalmology: Diseases of the Uveal Tract, vol IX. London: Henry Kimpton, 1966: 553-93.

24. Breger BC and Leopold IH: The incidence of uveitis in multiple sclerosis. Am J Ophthalmol 1966, 62: 540-5.

25. Porter R: Uveitis in association with multiple sclerosis. $\mathrm{Br} J$ Ophthalmol 1972, 56: 478-81.

26. Herbert J, Wilcox JN, Pham KTC, Fremeau RT Jr, Zeviani M, Dwork A, et al.: Transthyretin: a choroid plexus-specific transport protein in human brain. Neurology 1986, 36: 900-11.

27. Nathanson JA: $\beta$-Adrenergic-sensitive adenylate cyclase in secretory cells of choroid plexus. Science 1979, 204: 843-4.

28. Peraldi S, Dao BNT, Brabet Ph, Homburger V, Rouot B, Toutant M, et al:: Apical localisation of the alpha subunit of GTP binding protein $\mathrm{G} \alpha$ in choroidal and ciliated ependymocytes. J Neurosci 1989, 9: 806-14. 